\title{
Resistive or dynamic exercise stress testing of the pulmonary circulation and the right heart
}

\author{
To the Editor:
}

There has been recent revival of interest in exercise stress testing of the pulmonary circulation and the right ventricle $(\mathrm{RV})$ for the differential diagnosis of dyspnoea or latent pre- or post-capillary pulmonary hypertension [1-3]. However, guidelines remain cautious because of persistent uncertainties about protocols and the limits of normal [4]. In fact, exercise protocols still vary, with either cycling [3, 6-8], weight lifting $[5,6]$ or even handgrip [9] modalities, with invasive $[3,5-7,9]$ or non-invasive $[7,8,10]$ measurements either during [3, 6-10] or after [5] the exercise stress. Furthermore, pulmonary artery pressure (PAP) has been reported either alone $[5,8]$ or as a function of either cardiac output (CO) $[1-3$, $7,10]$, workload $[7,11]$ or oxygen uptake $\left(V^{\prime} \mathrm{O}_{2}\right)$ [12]. In the meantime, flow-corrected PAP during exercise emerges as the measurement of choice for pulmonary vascular function [1-3], and body position may not matter provided a sufficient number of exercise pressure-flow coordinates is generated to smoothen out higher baseline pulmonary vascular resistance (PVR) in the upright position [10], but there remains uncertainty about the most relevant measurements of $\mathrm{RV}$ function $[7,13,14]$. Although there is a rationale in favour of incremental dynamic exercise, there is currently no consensus about the optimal exercise modality. We therefore compared the cardiovascular and gas exchange effects of resistive (handgrip), mixed resistive/dynamic (weight lifting) or dynamic (cycling) exercise on the pulmonary circulation and the RV in healthy volunteers.

A total of 26 healthy young volunteers - 14 women and $12 \mathrm{men}$, height $170 \pm 10 \mathrm{~cm}$, weight $66 \pm 12 \mathrm{~kg}$, age $22 \pm 3$ years - gave informed consent to the study, which was approved by the Ethical Committee of Erasme University Hospital. All of them prospectively performed, in a random order, a handgrip at $40 \%$ of maximum voluntary contraction for $3 \mathrm{~min}$ or arm adduction lifting of dumbbells $(2.5 \mathrm{~kg}$ for women and $5 \mathrm{~kg}$ for men) for $3 \mathrm{~min}$, followed by cycling with the workload increased every 2 min (by $20 \mathrm{~W}$ in women and $30 \mathrm{~W}$ in men) until exhaustion. An echocardiography of the RV and the pulmonary circulation, as well as measurements of blood pressure (BP), heart rate (HR), ventilation $\left(V^{\prime} \mathrm{E}\right), V^{\prime} \mathrm{O}_{2}$ and carbon dioxide output $\left(V^{\prime} \mathrm{CO}_{2}\right)$, were performed at baseline and during the last minute of the handgrip and weight lifting and of each workload during cycle ergometry. The subjects waited between each of these exercise challenges until they felt rested and HR had returned to baseline. The exercise tests were performed in a semi-recumbent position in a dedicated echocardiography chair (Ergoselect 1200, Ergoline $\mathrm{GmbH}, \mathrm{Bitz}, \mathrm{Germany)}$ as previously reported [10].

Handgrip was performed using a dynamometer (Jamar Hydraulic hand dynamometer, Lafayette Instrument, Lafayette, IN, USA). Ventilation and gas exchange were measured using a metabolic system (CPX, Medgraphics, St Paul, MN, USA). Doppler echocardiography was performed with a portable ultrasound system (CX50 CompactXtreme Ultrasound System, Philips, Amsterdam, Netherlands).

PVR was calculated as [mean PAP (mPAP) - wedged PAP (PAWP)]/CO and total pulmonary vascular resistance (TPR) as $\mathrm{mPAP} / \mathrm{CO}$. RV-arterial coupling was indirectly assessed by the ratio of tricuspid annular plane systolic excursion (TAPSE) to systolic (s) PAP $[13,14]$ or by the ratio of sPAP to RV end-systolic area (ESA) [7].

@ERSpublications

Exercise stress testing of the pulmonary circulation and the right heart should be dynamic, not resistive http://ow.ly/ZEyF30c9RlP

Cite this article as: Motoji Y, Forton K, Pezzuto B, et al. Resistive or dynamic exercise stress testing of the pulmonary circulation and the right heart. Eur Respir J 2017; 50: 1700151 [https://doi.org/10.1183/ 13993003.00151-2017]. 
TABLE 1 Cardiopulmonary exercise test variables and echocardiography of the pulmonary circulation and the right ventricle at rest and at peak handgrip, weight lifting and cycle exercise tests in healthy subjects

\begin{tabular}{|c|c|c|c|c|c|}
\hline & Rest & Handgrip & $\begin{array}{l}\text { Weight } \\
\text { lifting }\end{array}$ & $\begin{array}{c}\text { Cycle ergometry } \\
\text { at AT }\end{array}$ & $\begin{array}{c}\text { Cycle ergometry } \\
\text { at peak }\end{array}$ \\
\hline Systolic BP mmHg & $106 \pm 15$ & $125 \pm 15^{*}$ & $139 \pm 27^{*}$ & $158 \pm 26 *$ & $180 \pm 34 * \pi$ \\
\hline HR beat. $\min ^{-1}$ & $75 \pm 15$ & $99 \pm 16^{*}$ & $101 \pm 18^{*}$ & $149 \pm 16 * \pi$ & $178 \pm 12 * 1++$ \\
\hline$V^{\prime} \mathrm{O}_{2} \mathrm{~mL} \cdot \mathrm{min}^{-1} \cdot \mathrm{kg}^{-1}$ & $4.6 \pm 0.8$ & $8.0 \pm 1.7^{*}$ & $12.1 \pm 3.3^{* \#}$ & $25.1 \pm 4.4 * \pi$ & $39.3 \pm 6.8^{* 1++}$ \\
\hline$V^{\prime} \mathrm{E} L \cdot \mathrm{min}^{-1}$ & $10 \pm 2$ & $18 \pm 5^{*}$ & $30 \pm 18^{* \#}$ & $51 \pm 13 * \pi$ & $103 \pm 27 * 1+$ \\
\hline$V^{\prime} E / V^{\prime} \mathrm{CO}_{2}$ slope & & $30.6 \pm 6.5$ & $30.4 \pm 5.7$ & & $31.7 \pm 2.9$ \\
\hline RER & $0.84 \pm 0.06$ & $0.94 \pm 0.13^{*}$ & $1.04 \pm 0.10^{* \#}$ & $0.99 \pm 0.02 *$ & $1.15 \pm 0.05 * \Re+$ \\
\hline$V^{\prime} \mathrm{O}_{2} / \mathrm{HR} \mathrm{mL}$-beat ${ }^{-1}$ & $4.2 \pm 1.7$ & $5.5 \pm 1.7^{*}$ & $8.1 \pm 3.0^{* \#}$ & $11.4 \pm 3.6$ *ף & $14.8 \pm 4.7^{* 9+}$ \\
\hline mPAP mmHg & $15.8 \pm 1.0$ & $18.5 \pm 1.5^{*}$ & $18.7 \pm 1.3^{*}$ & $26.3 \pm 3.7 * \pi$ & $28.8 \pm 3.2^{* 1++}$ \\
\hline $\mathrm{CO} L \cdot \mathrm{min}^{-1}$ & $5.6 \pm 1.1$ & $7.6 \pm 1.3^{*}$ & $8.1 \pm 1.6^{*}$ & $13.2 \pm 2.2 * \pi$ & $16.1 \pm 2.6 * 1+$ \\
\hline PAWP mmHg & $9.8 \pm 1.3$ & $10.5 \pm 1.4$ & $10.2 \pm 1.5$ & $12.5 \pm 1.8 * \pi$ & $12.9 \pm 1.7 * \pi$ \\
\hline PVR Wood unit & $1.1 \pm 0.5$ & $1.1 \pm 0.3$ & $1.1 \pm 0.4$ & $1.0 \pm 0.2$ & $1.0 \pm 0.1$ \\
\hline TPR Wood unit & $3.0 \pm 0.6$ & $2.5 \pm 0.4^{*}$ & $2.4 \pm 0.4^{*}$ & $2.0 \pm 0.2 * \pi$ & $1.8 \pm 0.1 * \pi+$ \\
\hline mPAP/CO slope & & $1.40 \pm 0.53$ & $1.27 \pm 0.61$ & & $1.16 \pm 0.10$ \\
\hline TAPSE mm & $25.6 \pm 2.1$ & $28.7 \pm 3.0$ & $29.6 \pm 2.7^{*}$ & $32.4 \pm 3.2 * \pi$ & $34.2 \pm 3.5^{* 91+}$ \\
\hline $\mathrm{S} \mathrm{cm} \cdot \mathrm{s}^{-1}$ & $14.1 \pm 1.9$ & $15.1 \pm 2.7$ & $16.1 \pm 2.8 *$ & $22.4 \pm 3.2 * \pi$ & $24.9 \pm 3.3^{* 9++}$ \\
\hline EDA $\mathrm{mm}^{2}$ & $18.0 \pm 3.1$ & $15.8 \pm 3.4^{*}$ & $15.2 \pm 3.0 *$ & $13.9 \pm 2.9 *$ & $12.2 \pm 2.0 *$ * \\
\hline ESA $\mathrm{mm}^{2}$ & $9.2 \pm 1.7$ & $7.3 \pm 1.6^{*}$ & $7.0 \pm 1.4^{*}$ & $5.8 \pm 1.2 * \pi$ & $4.9 \pm 0.9 * 91+$ \\
\hline FAC $\%$ & $48.8 \pm 2.3$ & $53.4 \pm 2.4^{*}$ & $53.0 \pm 3.0 *$ & $57.8 \pm 2.7^{*}$ & $59.8 \pm 3.7 * \pi$ \\
\hline TAPSE/sPAP $\mathrm{mm} \cdot \mathrm{mmHg}^{-1}$ & $1.13 \pm 0.10$ & $1.07 \pm 0.10$ & $1.08 \pm 0.09$ & $0.90 \pm 0.13^{\pi}$ & $0.79 \pm 0.09 * 9+$ \\
\hline $\mathrm{sPAP} / \mathrm{ESA} \mathrm{mmHg} \cdot \mathrm{cm}^{-2}$ & $2.54 \pm 0.47$ & $3.83 \pm 0.72^{*}$ & $4.07 \pm 0.88^{*}$ & $7.17 \pm 1.44 * \pi$ & $9.33 \pm 2.12^{* 1+}$ \\
\hline
\end{tabular}

AT: anaerobic threshold; $\mathrm{BP}$ : blood pressure; $\mathrm{HR}$ : heart rate; $V_{\mathrm{O}_{2}}^{\prime}$ oxygen uptake; $V_{\mathrm{E}}^{\prime}$ : ventilation; $V_{\mathrm{E}}^{\prime} / V^{\prime} \mathrm{CO}_{2}$ : ventilatory equivalent for carbon dioxide; RER: respiratory exchange ratio; mPAP: mean pulmonary artery pressure; CO: cardiac output; PAWP: wedged PAP; PVR: pulmonary vascular resistance; TPR: total pulmonary resistance; TAPSE: tricuspid annular plane systolic excursion; S: pulsed tissue Doppler tricuspid annulus S wave; EDA: end-diastolic area; ESA: end-systolic area; FAC: fractional area change; sPAP: systolic PAP. *: $p<0.05$ exercise versus resting; ${ }^{\#}$ : $p<0.05$ weight lifting versus handgrip; ${ }^{\text {n: }} p<0.05$ cycling at AT or at peak versus weight lifting; ${ }^{+}$: cycling at AT versus cycling at peak.

The results are presented as mean $\pm \mathrm{SD}$. The statistical analysis consisted of a repeated measures analysis of variance, with modified $\mathrm{t}$-tests for inter-group comparisons when the $\mathrm{F}$ ratio of the analysis of variance reached a critical p-value of $<0.05$ (MedCalc, version 16.4.3, MedCalc, Ostend, Belgium).

The main results of the different exercise tests are presented in table 1, which shows only resting and maximum exercise values for handgrip and weight lifting, and resting, ventilatory threshold and maximum exercise values for cycle ergometry and slopes of $\mathrm{mPAP}-\mathrm{CO}$ and $V^{\prime} \mathrm{E} / V^{\prime} \mathrm{CO}_{2}$.

Cycle ergometry was associated with highest $\mathrm{BP}, \mathrm{HR}, V^{\prime} \mathrm{O}_{2}, V^{\prime} \mathrm{E}$, respiratory exchange ratio (RER) and $\mathrm{O}_{2}$ pulse $\left(V^{\prime} \mathrm{O}_{2} / \mathrm{HR}\right)$, and a peak RER at 1.15 compatible with maximum exercise. Both handgrip and weight lifting were associated with increased $\mathrm{BP}, \mathrm{HR}, V^{\prime} \mathrm{O}_{2}$ and $\mathrm{O}_{2}$ pulse, but these changes were significantly smaller for handgrip than for weight lifting. RER increased with weight lifting only. There were no differences in $V^{\prime} \mathrm{E} / V^{\prime} \mathrm{CO}_{2}$ slopes.

The three exercise modalities were associated with increases in MPAP, CO, TAPSE, RV fractional area change (FAC) and sPAP/ESA, while PAWP increased only with cycling. PVR remained unchanged, but TPR consistently decreased from handgrip and weight lifting to cycling. The mPAP-CO slope tended to decrease from handgrip and weight lifting to cycling, but not significantly so. The tricuspid annulus S-wave increased with weight lifting and cycling only. RV end-diastolic area (EDA) and ESA decreased with the three exercise modalities. TAPSE/sPAP decreased with cycling and remained otherwise unchanged. All the haemodynamic, ventilatory and gas exchange variables that were markedly different during cycling compared to handgrip and weight lifting were already so at the ventilatory threshold. None of the variables differed between exercise modalities at a normalised $V^{\prime} \mathrm{O}_{2}$ (data not shown).

The marked superiority of cycling to handgrip and weight lifting as a cardiovascular and metabolic stress is shown in fig. 1, with mPAP as a function of CO and for TAPSE, S-wave and FAC as a function of $V^{\prime} \mathrm{O}_{2}$.

The present results are in keeping with the notion that static (handgrip) or predominantly resistive (weight lifting) exercise, in contrast to dynamic exercise (cycling), is associated with a much smaller increase in $V^{\prime} \mathrm{O}_{2}$ or $\mathrm{CO}$, but a proportionally larger increase in $\mathrm{BP}$ [15]. 

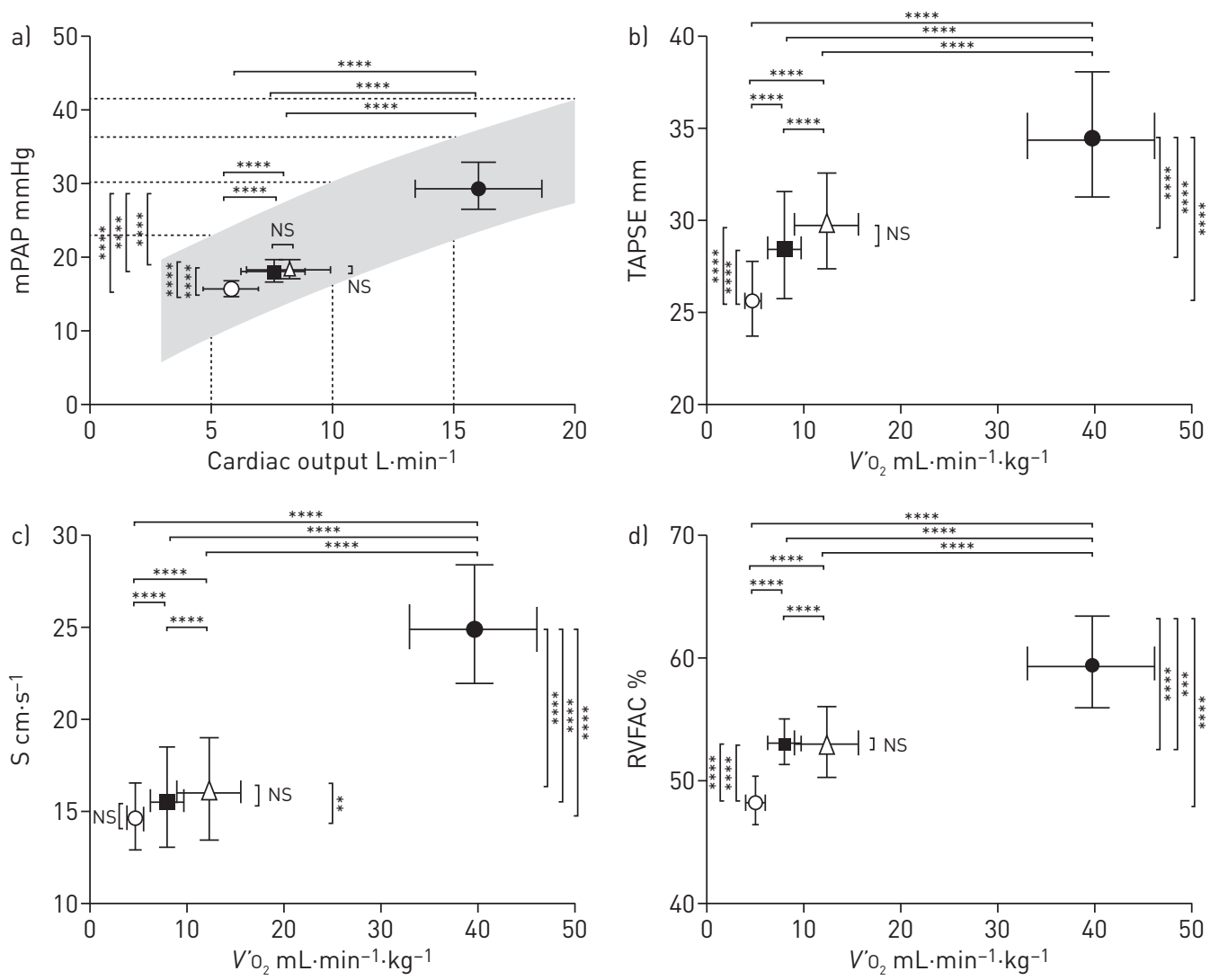

FIGURE 1 a) Mean pulmonary artery pressure (mPAP) as a function of cardiac output (CO), b) tricuspid annular plane systolic excursion (TAPSE) as a function of oxygen uptake $\left(V^{\prime} \mathrm{O}_{2}\right), c$ ) tricuspid annulus tissue Doppler velocity $\mathrm{S}$ as a function of $V^{\prime} \mathrm{O}_{2}$, d) right ventricular fractional area change (RVFAC) as a function of $V^{\prime} \mathrm{O}_{2}$ in healthy subjects before and during peak handgrip, weight lifting and cycling exercise tests. Vertical and horizontal bars indicate SD. The shaded area for MPAP-CO defines the limits of normal [1]. Cycling exercise is associated with much larger changes in mPAP, TAPSE, $S$ and FAC during cycling than during handgrip or weight-lifting exercise modalities. ${ }^{*}: p<0.05,{ }^{* *}: p<0.01,{ }^{* * *}: p<0.001,{ }^{* * * *}: p<0.0001$.

Resting and cycling exercise echocardiographic measurements of the pulmonary circulation and the RV were of the same magnitude as previously reported $[1-3,13,14]$; in particular, mPAP-CO relationships were similar to those reported previously in noninvasive as well as invasive studies [1-3]. The slopes of mPAP-CO were somewhat higher during handgrip or weight lifting than during cycling, but this is related to the slight natural curvilinearity of these relationships resulting in a relatively smaller increase in $\mathrm{mPAP}$ at higher $\mathrm{CO}[1,2]$.

One could wonder if more strenuous handgrip or weight lifting at a higher \% of maximum voluntary contraction would have been more challenging. However, in the present experiments, such higher workloads were not tolerated long enough to allow for the echocardiographic examination. In fact, handgrip testing was previously reported to increase CO by $26 \%$ with upper limits of normal PAP in patients [9]. This is comparable to a $36 \%$ increase found in the present study in younger and healthy adults. Weight lifting was previously shown to increase CO and PAP by $52 \%$ and $26 \%$, respectively, in scleroderma patients with normal haemodynamics [5]. This compares with the increases in CO and mPAP by $45 \%$ and $18 \%$, respectively, in the present study.

The limits of normal of exercise stress testing of the pulmonary circulation are now reasonably well defined, with an upper limit of $3 \mathrm{mmHg} \cdot \mathrm{L}^{-1} \cdot \mathrm{min}^{-1}$ for the slope of a multipoint $\mathrm{mPAP}-\mathrm{CO}$, or a generally agreed upon maximum exercise TPR $[1,2]$. It may be important to use an exercise modality associated with the largest increase in CO, as TPR decreases with increasing CO [1]. Thus, falsely abnormal responses could be suspected to occur in the case of a TPR $>3 \mathrm{mmHg} \cdot \mathrm{L}^{-1} \cdot \mathrm{min}^{-1}$ at a CO largely below $10 \mathrm{~L} \cdot \mathrm{min}^{-1}$.

The limits of normal of the indices of RV function during exercise are less well known. The increases in sPAP/ESA and decreases in TAPSE/sPAP found with cycling in the present study agree with previously reported values $[7,14]$. Large increases in TAPSE, S (tricuspid annulus tissue Doppler velocity), FAC and 
sPAP/ESA during cycle ergometry again point at this purely dynamic maximum exercise test as being preferable for exercise stress testing of the RV. The functional significance exercise-induced decline in TAPSE/sPAP may not be entirely clear. Previously reported good agreement between sPAP/ESA by echocardiography and sPAP/end-systolic volume by magnetic resonance imaging suggests that this variable might be a valid estimate of RV contractile reserve [7].

An important limitation of this study was in the use of Doppler echocardiography to evaluate the pulmonary circulation and the RV, instead of gold-standard invasive measurements and magnetic resonance imaging as previously reported [7]. However, the marked differences in haemodynamics and RV function during cycling compared with handgrip and weight lifting are unlikely to be accounted for by insufficient methodology. It remains that exercise stress echocardiography needs more validation, with rigorous assessments of both accuracy and precision, as previously reported at rest [16]. Another limitation of this study is that the different exercise modalities were investigated in healthy subjects, so the results may not be directly transposable to patients. However, the contrasted responses to resistive, resistive/dynamic and dynamic exercise strongly argue in favour of the latter for exercise stress testing of the pulmonary circulation and the RV and offer a potentially useful estimate of the limits of normal.

Yoshiki Motoji $^{1,2}$, Kevin Forton ${ }^{1}$, Beatrice Pezzuto ${ }^{1}$, Vitalie Faoro ${ }^{1}$ and Robert Naeije ${ }^{1,2}$

${ }^{1}$ Laboratory of Exercise Physiology, Faculty of Motor Science, Université Libre de Bruxelles, Brussels, Belgium.

${ }^{2}$ Department of Cardiology, Erasmus University Hospital, Brussels, Belgium.

Correspondence: Dr R. Naeije, Department of Pathophysiology, Erasmus Campus, CP 604, Route de Lennik, 808, B-1070 Brussels, Belgium. E-mail: rnaeije@ulb.ac.be

Received: Jan 212017 | Accepted after revision: March 292017

Conflict of interest: Disclosures can be found alongside this article at erj.ersjournals.com

\section{References}

1 Lewis GD, Bossone E, Naeije R, et al. Pulmonary vascular hemodynamic response to exercise in cardiopulmonary diseases. Circulation 2013; 128: 1470-1479.

2 Naeije R, Vanderpool R, Dhakal BP, et al. Exercise-induced pulmonary hypertension physiological basis and methodological concerns. Am J Respir Crit Care Med 2013; 187: 576-583.

3 Herve P, Lau E, Sitbon O, et al. Criteria for diagnosis of pulmonary hypertension at exercise. Eur Respir J 2015; 46: 728-737.

4 Galiè N, Humbert M, Vachiery J-L, et al. 2015 ESC/ERS Guidelines for the Diagnosis and Treatment of Pulmonary Hypertension. Eur Respir J 2015; 46: 903-975.

5 Steen V, Chou M, Shanmugam V, et al. Exercise-induced pulmonary arterial hypertension in patients with systemic sclerosis. Chest 2008; 134: 146-151.

6 Borlaug BA, Nishimura RA, Sorajja P, et al. Exercise hemodynamics enhance diagnosis of early heart failure with preserved ejection fraction. Circ Heart Fail 2010; 3: 588-595.

7 Claessen G, La Gerche A, Voigt JU, et al. Accuracy of echocardiography to evaluate pulmonary vascular and right ventricular function during exercise. JACC Cardiovascular Imaging 2015; 9: 532-543.

8 Grünig E, Weissmann S, Ehlken N, et al. Stress Doppler echocardiography in relatives of patients with idiopathic and familial pulmonary hypertension : results of a multicenter European analysis of pulmonary artery pressure response to exercise and hypoxia. Circulation 2009; 119: 1747-1757.

9 Schwaiblmair M, Faul C, von Scheidt W, et al. Detection of exercise-induced pulmonary arterial hypertension by cardiopulmonary exercise testing. Clin Cardiol 2012; 35: 548-553.

10 Forton K, Motoji Y, Deboeck G, et al. Effects of body position on exercise capacity and pulmonary vascular pressure-flow relationships. J Appl Physiol 2016; 121: 1145-1150.

11 Lewis GD, Murphy RM, Shah RV, et al. Pulmonary vascular response patterns during exercise in left ventricular systolic dysfunction predict exercise capacity and outcomes. Circ Heart Fail 2011; 4: 276-285.

12 Tolle JJ, Waxman AB, Van Horn TL, et al. Exercise-induced pulmonary arterial hypertension. Circulation 2008; 118: 2183-2189.

13 Guazzi M, Bandera F, Pelissero G, et al. Tricuspid annular plane systolic excursion and pulmonary arterial systolic pressure relationship in heart failure: an index of right ventricular contractile function and prognosis. Am J Physiol Heart Circ Physiol 2013; 305: H1373-H1381.

14 D'Alto M, Pavelescu A, Argiento P, et al. Echocardiographic assessment of right ventricular contractile reserve in healthy subjects. Echocardiography 2016; 34: 61-68.

15 Kivowitz C, Parmley WW, Donoso R, et al. Effects of isometric exercise on cardiac performance. The grip test. Circulation 1971; 44: 994-1002.

16 D'Alto M, Romeo E, Argiento P, et al. Accuracy and precision of echocardiography versus right heart catheterization for the assessment of pulmonary hypertension. Int J Cardiol 2013; 168: 4058-4062. 